\title{
Téoros
}

Revue de recherche en tourisme

\section{Le Médiathel : un nouveau concept de gestion touristique}

\section{Dominique Alunni et Robert Lanquar}

Volume 5, numéro 3, novembre 1986

La gestion touristique : les nouvelles technologies

URI : https://id.erudit.org/iderudit/1080536ar

DOI : https://doi.org/10.7202/1080536ar

Aller au sommaire du numéro

Éditeur(s)

Université du Québec à Montréal

ISSN

0712-8657 (imprimé)

1923-2705 (numérique)

Découvrir la revue

Citer cet article

Alunni, D. \& Lanquar, R. (1986). Le Médiathel : un nouveau concept de gestion touristique. Téoros, 5(3), 19-43. https://doi.org/10.7202/1080536ar d'utilisation que vous pouvez consulter en ligne.

https://apropos.erudit.org/fr/usagers/politique-dutilisation/ 
Un Médiathel fonctionne depuis le début 1986 à Poix-de-Picardic (Somme). Le Médiathel est un concept qui a été lancé par I'U.P.C.S. Communication et Développement. Il s'agit du premier hôtel d'une chaîne nouvelle avec une ouverture sur la technologie et la communication en collaboration avec les partenaires locaux. C'est le premier maillon d'une chaine d'hôtels-restaurants d'application qui auront pour but de favoriser le developpement local et de permettre une formation interactive aux métiers du tourisme et de l'hôtellerie liće à une pédagogie de l'action.

Depuis la création du Centre de Culture Ouvrière (C.C.O.) en 1945, puis de l'Institut national de formation des animateurs de collectivités (INFAC) en 1963, de CREAR en 1974 et de l'TNFATH (Institut national de formation aux métiers du tourisme et de 1'hobtellerie) en 1977, une culture s'est créée et a été diffusce, des formateurs aux formés, autour des concepts de développement socio-économique local. d'esprit de pluralisme respectant la personne et laissant à chacun le choix de ses engagements, de recherche d'innovations techniques et culturelles, de communication dans tous les sens du terme.

La création de l'U.P.C.S. (Communication et Développement), en 1983, n'a fait que renforcer ces options. Cette culture peut être résumée dans le concept de la "pédagogie de l'action" faisant alterner des sessions theoriques en groupe et des stages pratiques en entreprises ou sur le terrain avec trois axes.

- celui de l'éducation des travailleurs à travers l'expérimentation personnelle empirique (pratique sanctionnée par l'acquis du savoir-faire).

- celui de la culture artisanale et artistique, culture de la "main", de la technique pour favoriser l'épanouissement des hommes au sein de leurs collectivités.

\footnotetext{
"Experts intemationaux du tourisme MM. Dominicaue Alunni et Robert Languar sont, respectivement, president ot directeur des ótudas de la firme de recherche INFAC.
}

- celui des sciences sociales pour favoriser la communication et l'animation des individus et des groupes au travail ainsi que dans leur cadre de vie.

L'U.P.C.S. possède, en effet, l'experrience, les equipements, les hommes qui lui permettent de positionner de manière ordonnée et cohérente ses formations. Mais il lui devenait nécessaire de les présenter avec une unité â l'extérieur pour gagner le pari d'être le principal producteur de formation supérieure de l'économie sociale en Franoe.

Comme l'entreprise est au coeur du projet de l'U.P.C.S., c'est-di-dire le lieu de rencontre des facteurs de production avec des hommes et des femmes vivant dans des collectivités de vie et de travail, la formation doit avoir pour objectif d'améliorer leurs competences et leur cadre de vie et de travail ât travers une pratique d'épanouissement personnel. Par ailleurs, I'U.P.C.S. veut s'intégrer dans un monde de plus en plus interdépendant où les entreprises comme les hommes traversent les frontieres de plus en plus souvent pour exercer leurs activités, Le Médiathel est une nouvelle entreprise de communication, de développement et de formation proposée par l'Union pour tenir compte de l'importance de ces évolutions, en particulier pour les entreprises du tourisme, de thotellerie et de la communication économique, sociale et culturelle, c'est-à-dire de tous les métiers lies à la rencontre (animation, compunication, vojages, vacances, etc...). selon deux directions concomitantes, alternées ou simultanées, de formation:

- traditionnelle aux missions de gestion/administration et de communication (savoir gérer, savoir vendre, savoir développer, savoir communiquer), avoc aussi une formation à l'animation et à la communication ( $y$ compris les langues etrangères).

- technologique pour faciliter la connaissance et la maîtrise technique des savoirs dans l'hotellerie, la restauration et la cuisine, l'aménagement physique du tourisme, la télématique et l'informatique. Pour cette partie technologique, it s'agit de former à:
+ une culture technologique générale autant qu'ćconomique pour parvenir à la mấtrise d'une ingénierie des domaines concernés et savoir s"adapter à l'évolution technologique et économique;

+ la maîtrise et l'apprentissage des savoirfaire technologiques de haut niveau lices au développement de l'hótellerie, de la restauration et de la cuisine, de la communication audio-visuelle et médiatique;

+ une polycompétence pour les cadres et le personnel de maîtrise qui facilitera une attitude pédagogique vis à vis du personnel (les cadres et agents de maîtrise sont dans un sens aussi les formateurs du personnel des entreprises). Ils doivent done pouvoir benćficier d'une formation de formateurs.

\section{Les partenaires du Médiathel}

Forte de ces concepts 1'U.P.C.S. s'est attaché a faire évoluer le cadre de la formation en application. La formation doit en effet servir de catalyseur au développement local. Des partenaires ont êté convaincué du bien-fondé de cette attitude. Pour le premier Médiathel, celui de Poix-en-Picardie, trois partenaires sont associés: les collectivités locales (Conscil Régional, Conseil Général, Municipalité), une Banque (le Crédit Agricole) et une des composantes de I'U.P.C.S., l'INFATH (Institut National de formation professionnelle aux métiers du tourisme et de I'hôtellerie) dans une mission de développement local à partir d'un hôtel rural acheté par la municipalité en 1983 et rénové par l'INFATH.

\section{Les directions d'action}

Quatre directions ont été définies par le Médiathel:

- c'est un équipement hôtelier contemporain pouvant accueillir une clientele de passage et des groupes en séminaire, disposant d'un restaurant gastronomique (cuisine régionale, mais aussi diététique) et d'un bar-restaurant avec cuisine rapide. 
- c'est ensuite un pôle d'animation. notamment avec son bar ouvert en permanence qui permet la diffusion, à partir d"un centre micro-serveur, d'informations télématiques sur les activités locales (on trouve en effet sur les Minitels* des informations culturelles, économiques, touristiques et sportives, le bulletin municipal, des informations pratiques sur les trains et autobus, les services de sécurité, le calendrier des fêtes...). Le Médiathel possède aussi une antenne parabolique qui permet de suivre 17 programmes européens de télévision (même dans les chambres) et une chaîne intérieure. En outre, le Médiathel est en relation avec I'ANIT (Agence nationale pour l'information touristique), non seulement pour sa propre promotion, mais surtout pour la création d'informations sur des produits et circuits locaux. Le Médiathel est aussi un pöle d'animation, avec des salles polyvalentes pouvant accueillir des vidéoprojections et des manifestations ponctuelles organisées par les collectivités locales et les associations. Il faut compter au moins une dizaine de salles polyvalentes de réunions pour un Médiathel de 60 lits.

- c'est encore et surtout un Centre de formation et d'orientation pour adultes. Le Médiathel accueille en permanence les formations professionnelles de longue et de courte durées pour les métiers de l'hôtellerie, du tourisme et de la conmunication tout en suivant l'ếvolution de ces métiers.

- c'est enfin un Centre de conseil aux entreprises (gestion, équipement, développement) et d'aide à la création. Selon les besoins de l'́́conomie, ces formations peuvent être complétées et diversifices: crétion d'entreprises. management et gestion, informatique... Conçu et développé avec le concours des collectivités locales, le Médiathel favorise la présence de clientèles nouvelles et contribue à l'animation de la Ville et à l'essor du commerce et du tourisme. II devient ainsi un véritable pôle de communication et de développement local.

\section{La taille optimale d'un Médiathel}

Pour une petite ville comme Poix-enPicardie située sur une ancienne route de circulation intense entre la France et la Grande-Bretagne, il fallait partir de rien. Les ćtudes de marché avaient seulement montré que pour la région de Picardie, le besoin en séminaires de formation et ceux d'autres clientèles entrainerait la nécessité de réaliser un équipement d'hébergement d'une taille optimale, pour le moment de 35 lits avec des possibilités d'extension.

Mais, il aurait été difficile à partír des chif- fres de la demande touristique à Poix-enPicardie d'établir une part de marché à conquérir. Ce n'est pas l'objectif d'un Médiathel qui cible d'autres clientèles que la clientèle traditionnelle de I'hôtellerie auquel il devrait être complémentaire et apporter une clientèle supplémentaire si ses gestionnaires sont capables d'attirer des séminaires importants. C'est pourquoi il faut utiliser une autre approche, celle de l'offre, pour déterminer la taille du futur Médiathel en considérant trois facteurs:

- la taille optimale d'un Módiathel qui fonctionnerait dans de bonne conditions d'efficacité et d'équilibre de gestion.

- le pourcentage de capacité supplémentaire qu'il entraînerait pour la communauté locale d'accueil (il ne devrait pas dans un premier temps dépasser $25 \%$ de la capacité existante pour ne pas avoir un impact négatif à court terme).

- la taille des equipements et des hébergements complémentaires de la localité dans lequel il est situé.

\section{La catégorie optimale}

Étant donné la clientèle ciblée, il s'agit que le Médiathel soit d'un bon confort (type $2^{\circ}$ ) comportant:

- un hall de réception,

- une entrée de l'hôtel indépendante, puisqu'il y a également un restaurant et un bar-restaurant,

- un chauffage central intégré,

- un equipement sanitaire (eau chaude et froide à toute heure),

- un téléphone intérieur et avec le réseau dans toutes les chambres

- le cablage et la possibilité d'installer une télévision à la demande,

- la liaison avec un centre serveur télématique sur Minitel,

- une antenne parabolique et un répartiteur électronique de programmes,

- une occultation opaque extérieure,

- un revêtement du sol,

- un bon confort acoustique,

- une surface utile minimum par chambre, sanitaires non compris de 8 mètres carrés pour une chambre 1 personne et 9 mètres carrés pour une chambre 2 personnes,

- des sanitaires privés pour chaque chambre et des water-closets particuliers en local sanitaire clos.

- des water-closets communs près du hall, des salles de réunions et séminaires et du restaurant et bar.

- équipement électrique des chambres et sanitaires,

- équipements électriques minimum des locaux communs,

- un personnel de réception parlant au moins 1 langue étrangère,

- petit dejeûner pouvant être servi dans les chambres.

- restauration.
- parking,

- une buanderie.

Les tarifs journaliers pouvaient être en pension complette de l'ordre de 300FF (50\$), ce qui ne concurrence en aucune manière les hôtels 2* des environs.

\section{Les activités}

En peu de temps, le Médiathel est devenu un pole d'animation pour Poix-en-Picardie. Les responsables commerciaux se sont en effet rendus compte des énorme possibilités de la région en matière d'agroalimentaire et d'artisanat. Grace au Mediathel, la première Foire gastronomique a pu être lancé en Picardie (septembre 1986) et une fête de l'artisanat organisée dans un village voisin, Conty. Avec le Crédit Agricole, ils ont même été à l'origine d'un spec tacle "son et lumierre" sur la Picardie, dans un autre site avoisinant, Ailly-sur-Noye, où plus de 250 acteurs-amateurs de la région ont pu évoluer et attirer jusqu'à 2600 personnes par spectacle.

En outre, le Médiathel a des objectifs d'animation intérieure. Il comporte des salles polyvalentes pouvant accueillir des vidéoprojections et des manifestations ponctuelles.

Pour un Médiathel de 60 chambres, il semble qu'une bonne douzaine de salles soient nécessaires, de $25 \mathrm{~m}^{2}$ à $200 \mathrm{~m}^{2}$.. soit un total de $600 \mathrm{~m}^{2}$ de salles polyvalentes. Si, à certains moments de l'anné, des salles manquent, il faut négocier avec des hôteliers locaux ou d"autres organisations et associations la possibilité de location.

Ensuite, étant donné le type de clientèle, il faut prévoir un bar-restaurant et un réstaurant avec des possibilités d'une part gastronomique, d'autre part diététique. $\mathrm{Ce}$ restaurant est aussi un restaurant d'application avec une spécialité comme la cuisine sous vide, ce qui permet une production locale et une commercialisation. La cuisine centrale est donc un des lieux clés de tout le complexe: cuisine d'application et de formation.

L'animation du bar est assurée, entre autres, par le centre serveur sur Minitel. Il est utilisé pour connaitre les informations locales culturelles, économiques, touristiques et sportives. Il peut servir de messagerie. Il est au coeur de l'information du Médiathel, on y rouvera aussi les menus et la carte du jour, que l'état du ciel, la réservation des courts de tennis, la température de la piscine la plus proche, le calendrier des événements et manifestations, etc...

La part du matériel informatique et audio visuel dans l'investissement total représente moins de $5 \%$ du total. En effet, dans le premier Médiathel, celui de Poix-en-Picardie,

\footnotetext{
+ le centre serveur est revenu à $200,000 \mathrm{FF}$,
} 
Le ministère du Tourisme honore six lauréats nationaux lors de la remise des grands prix du tourisme

Le deuxième gala annuel des Grands Prix du Tourisme Québecois, qui s'est déroulé au Palais des Congrès de Montréal a permis a plus de 1000 personnalités de l'industrie touristique d'applaudir les réalisations des lauréats provenant des 17 régions du Québec. Le ministre du Tourisme du Québec, M. Picotte a profité de cette cérémonie de gala pour souligner que: "cette manifestation se veut un geste de reconnaissance, non seulement pour ceux qui se sont signalés d'une façon particulière, mais aussi pour tous les intervenants sans exception."

Il a poursuivi en disant: "Qu'il est fort encourageant pour un ministre de pouvoir compter sur autant de gens, qui avec conviction et acharnement, contribuent à la vitalitế du secteur tourisme.

M. Picotte a conclu en felicitant les six gagnants nationaux et en les assurants: "que ce couronnement n'est qu'un reflet de l'honneur qui leur échoit" ".

L'hotel La Sapinière de Val-David a remporté le prix de l'excellence touristique, le summum des prix décernés par le ministềre, pour sa contribution exceptionnelle dans le domaine hôtelier.

La Sapinière est un hôtel de 75 chambres, situé dans un cadre enchanteur au coeur des Laurentides et appartient à la famille de $\mathrm{M}$. Jean Louis Dufresne.

L'entreprise fête son 50 ième anniversaire cette année et elle est l'un des premiers hôtel au Canada à faire partie du regrou- pement d'établissement de qualité "Relais et Châteaux"'.

Le prix de l'excellence a été décerné par le président d'honneur de ce deuxième gala annuel, le ministre du Tourisme Yvon Picotte.

Outre La Sapinière, cing autres entreprises sélectionnées parmi 74 finalistes régionaux ont été proclamés "lauréats nationaux" pour les meilleures performances dans les 5 catégories considérées comme etant les moteurs de l'essor touristique: création de nouveaux produits, développement des ressources, comportements et structure d'accueil, promotion des produits et des destinations et développement d'événements à incidence touristique.

Pour qu'une performance soit jugete remarquable, il faut qu'elle ait, selon la taille et la capacité du candidat, contribué de façon visible et rentable au développement de l'image et du produit touristique québécois.

Le Musée des Beaux-Arts de Montréal a mérité un des trophées nationaux du tourisme québécois pour avor présenté l'évếnement de l'année soit l'Exposition Picasso. Le nouveau Maire de Montréal, M. Jean Doré, qui faisait sa première sortie depuis son assermentation, a remis le trophée aux dirigeants du Musée. Les autres lauréats honorés pour leurs contributions remarquables et exceptionnelles à mettre en valeur le produit touristique sont: dans la catégorie accueil touristique, Les Entreprises Stoneham (région Québec), dans la catégorie développement touristique, Hôtel Le Chanteclerc (région des Laurentides), dans la catégorie promotion touristique, Le carnaval de Québec et les Excursions et expéditions de canots des lles de Sorel inc. (région de la Montérégie) dans la catégorie innovation touristique.

\section{Le ministre rend public un premier bilan}

Parallèlement à cela, le ministre du Tourisme a rendu public les principales données statistiques sur le nombre de touristes venus au Québec au cours des huits premiers mois de l'annee 1986. Cette annonce arrive à point alors qu'on est à encourager et récompenser, par les Grands Prix du Tourisme, les efforts de tout(es) ceux et celles qui se sont particulièrement signalés dans le milieu touristique.

Donc par rapport à l'année précédente, $\mathrm{M}$. Picotte souligne une nette augmentation de $7 \%$ de visiteurs américains et ce, malgré I'attrait qu'a exercé l'exposition universelle de Vancouver. On souligne également une stabilitế des départs vers les E.U., alors que les départs vers les autres pays ont diminué de près de $5 \%$. L'annonce de cette heureuse nouvelle vient donc s'ajouter aux actions d'encouragement engendres par les Grands Prix du Tourisme.

Les Grands Prix du Tourisme sont aussi important pour l'industrie touristique que le sont les Awards pour l'industrie du spectacle puisque l'activité économique qu'est le tourisme soutient plus de 120000 emplois et produit un impact de l'ordre de 3050 milliards de dollars.

Les Grands Prix du Tourisme québécois constituent une occasion privilégié de perfectionnement et de promotion pour les régions, les intervenants et les produits touristiques. $f$

\section{Marie-Claude Cardin}

\section{Le Médiathel}

(Suite de la page 20)

+ les logiciels informatiques et la matériel aे $300,000 \mathrm{FF}$ (micro-ordinateurs à la disposition de la clientèle qui peut le louer et celui de l'exploitation).

+ le système audio-visuel à $300,000 \mathrm{FF}$ (c'est-à-dire une antenne parabolique, un décodeur, un grand écran, une camera scop-mobile, une sono mobile et un vidéo). Il faudra y ajouter le coult d'un répartiteur.

Cet équipement peut recevoir un financement différent du Médiathel, étant donné son impact. S'il sert la communauté d'accueil dans sa politique d'information touristique, une subvention $\mathrm{d}^{\prime}$ equipement peut être trouvée.

En ce qui concerne l'animation extérieure, le Médiathel devrait être à la pointe d'une animation fondéc sur des produits nouveaux, en particulier en matière de santé. Par exemple, un des futurs Médiathels, actuellement en projet, pourra compter d'une part sur un centre de maintien et de santé (salle de gymnastique et kinésibalnéothérapie), d'autre part sur des équipements sportifs communs à tous les usagers, y compris un parcours de santé et une piscine.

\section{Les limites du développement des Médiathel}

Il ne fallait pas que le futur établissement soit taxé de paracommercialisme par les professionnels locaux du tourisme, plus spécifiquement par les hoteliers des environs. Ceux-ci doivent voir dans le futur complexe, un lieu de développement local qui va amener une clientèle à des événements collectifs (groupes sportifs, séminaires et réunions, etc...).

La taille de l'établissement à créer doit done être seulement déterminée par les besoins en séminaires de formation et du développement potentiel de cet organisme et des accords qu'il pourra faire avec d'autres organes de formation.

Différentes formules sont été envisagées. Mais la création d"un établissement hôtelier "Hôtel de Tourisme classé 2 étoiles" comme etablissement à caractère commercial, appartenant à un propriétaire unique et géré par un concessionnaire exploitant semble la plus simple et la plus fiable. a la fois pour le propriétaire qui pourra céder cet hôtel en bail emphytéotique à un gestionnaire sur 30 ou 40 ans et pour le gestionnaire qui sera assuré d'une certaine pérennité dans son administration. Il existe en effet en France des exemples de plus en plus nombreux où une société à vocation commerciale a pu être créée par des collectivités locales et organismes collectifs et sociaux. Cette voie semble d'ailleurs être une des solutions qui permettront au secteur français du tourisme social et associatif de s'intégrer dans le secteur commercial tout en ne perdant pas leur âme de "service public" pour leurs membres et à la collectivité. $f$

Nogent-sur-Marne, le 6 octobre 1986 